\title{
Utilización y accesibilidad a medicamentos en el Sistema Nacional del Seguro Social de Argentina
}

José F. Parodi', Teodoro J. Oscanoa², Miguel A. Fernández- Pastor ${ }^{3}$, Maria Cecilia Garbarino ${ }^{4}$, Mauro Tomas Ghersi ${ }^{5}$

\section{RESUMEN}

Objetivo: Investigar la utilización y accesibilidad a los medicamentos en pacientes cubiertos por el Sistema Nacional del Seguro Social de Argentina (SNSSA).

Material y Métodos: Se estudió la accesibilidad a los medicamentos que eran consumidos los últimos 15 días antes de ser hospitalizados, en 500 pacientes en 5 hospitales en el área de Buenos Aires.

Resultados: Del total de encuestados, 292 pacientes tuvieron prescripción médica 15 días antes de ser hospitalizados, 171 $(58,6 \%)$ pacientes no tuvieron accesibilidad a medicamentos. La media de edad fue: $47,4+18,8$ y rango de 0.1 a 89 años; $173(34,6 \%)$ fueron de sexo masculino y $327(65,4 \%)$ femenino. La distribución de la cobertura de los pacientes estudiados fue predominantemente perteneciente al Sistema Público con 280 (56,0\%), Obra Social 175 (35,0\%) y Prepago $45(9,0 \%)$. Las razones de la no accesibilidad, fueron principalmente: errores en los procesos que van desde la emisión de las recetas, Ilenado incorrecto de los formularios hasta la no disponibilidad de los medicamentos en las farmacias.

Conclusiones: La falta de accesibilidad a medicamentos en pacientes cubiertos por el SNSSA es un fenómeno frecuente.

(Horiz Med 2014; 14(2): 11-17)

Palabras clave: Accesibilidad a los Servicios de Salud, Utilización de Medicamentos. Política Nacional de Medicamentos, Política Nacional de Asistencia Farmacéutica. (Fuente: DeCS BIREME)

\section{Medicine access and utilization in a population covered by National Social Security System of Argentina}

\begin{abstract}
Objective: To investigate the use and accessibility to drugs in patients covered by the National Social Security Argentina (SNSSA).

Material and Methods: We studied the accessibility to drugs that were consumed the last 15 days before hospitalization, in 500 patients in 5 hospitals in the area of Buenos Aires.

Results: Of the 500 patients surveyed, 292 patients had medical prescription 15 days before being hospitalized, 171 (58.6\%) patients had no access to medicines. The mean age was $47.4+18.8$ and range of 0.1 to 89 years; 173 (34.6\%) were male and $327(65.4 \%)$ female. The distribution of coverage of patients studied was predominantly belonging to the Public System with $280(56.0 \%)$, Social Work 175 (35.0\%) and Prepay 45 (9.0\%). The reasons for non- accessibility were mainly: the errors in processes ranging from issuing prescriptions, improper filling of forms to non-availability of medicines in pharmacies. Conclusions: The lack of access to medicines for patients covered by SNSSA is a common phenomenon. (Horiz Med 2014; 14(2): 11-17)
\end{abstract}

Key words: Accessibility of Health Services, Drug Utilization. National Drug Policy, National Pharmaceutical Assistance Policy. (Source: MeSH NLM)

1 Director del Centro de Investigación del Envejecimiento (CIEN) de la FMH. de la USMP. Lima Perú. Magister en Salud Pública con Mención en Epidemiología. Especialista en Geriatría.

2 Miembro del CIEN de la FMH. de la USMP. Lima Perú. Doctor en Medicina, Especialista en Medicina Interna.

3 Director del Centro Interamericano de Estudios de Seguridad Social (CIESS). Buenos Aires, Argentina.

4 Medico General. Universidad de Buenos Aires de Argentina (UBA)

5 Estudiante de medicina.Universidad de Buenos Aires de Argentina (UBA) 


\section{INTRODUCCIÓN}

Uno de los aspectos esenciales de la evaluación de la calidad de un sistema de salud, es la accesibilidad a medicamentos, ya que una de las principales intervenciones terapéuticas en medicina, es la prescripción farmacológica. La expedición de una receta a un paciente, es solo el inicio de una serie de procesos que debería terminar exitosamente con la adquisición del fármaco y la mejoría o curación de la enfermedad, el objetivo final.

Es esencial que el fármaco llegue al paciente; las investigaciones destinadas a estudiar los factores que pueden afectar este proceso, son denominados estudios de accesibilidad de medicamentos, definiéndose ésta como: la obtención con o sin pago directo por el usuario del medicamento recetado (1). Por otro Iado, los Estudios de Utilización de Medicamentos (EUM), son investigaciones que analizan la comercialización, distribución, prescripción y uso de fármacos en una sociedad, haciendo énfasis especial en las consecuencias médicas, económicas y sociales de este uso. El objetivo principal de un EUM es lograr la mejor atención posible en el cuidado de la salud mediante un uso racional de los medicamentos (2).

El sistema de salud en Argentina, tiene tres bases fundamentales: el público, con financiación y provisión pública; el de seguridad social (sistema de obras sociales), y el sistema de seguro voluntario prepago, que se organiza según los cálculos de riesgo actuarial (3).El sistema de Seguridad Social en Argentina, está compuesto por los siguientes seis componentes: el régimen previsional; las obras sociales; el seguro de desempleo; sistema de riesgos del trabajo; el PAMI (Programa de Atención Médica Integral), que brinda cobertura médica a la tercera edad; y el régimen de asignaciones familiares, que otorga subsidios múltiples (4). La cobertura de salud, tiene dos subsistemas principales: el de Obras Sociales, para los trabajadores en actividad y el Instituto Nacional de Servicios Sociales para Jubilados y Pensionados (INSSJyP) o PAMI (Programa de Atención Médica Integral) (5). Las Obras Sociales son las principales organizaciones de la Seguridad Social Médica en la Argentina, cubriendo aproximadamente $50 \%$ de la población incluidos los familiares y adherentes. La fuente de financiamiento es por aportes de los trabajadores y empleadores, y una parte proviene del presupuesto general (en el caso del Instituto Nacional de Servicios Sociales para Jubilados y Pensionados-PAMI). Muy pocas entidades poseen capacidad instalada propia, siendo mayoritariamente instituciones financieras que contratan servicios con el sector privado y esporádicamente con hospitales públicos (6). El copago es variable según la Obra Social o empresa que se elija, y generalmente es una cantidad fija del valor de la consulta o estudio. Las prescripciones se hacen sólo por producto genérico o marca comercial dentro de listados autorizados. El farmacéutico puede cambiar la marca del medicamento (7).

El presente estudio, tuvo como obj etivo describir las características de la utilización de medicamentos y su accesibilidad en pacientes cubiertos por el Sistema Nacional de Seguro Social de Argentina.

\section{MATERIAL Y MÉTODOS}

Estudio de tipo observacional, de corte transversal. Se llevó a cabo del 1ro de Noviembre del 2010 al 30 de Marzo del 2011, en 500 pacientes hospitalizados en 5 hospitales, en el área de Buenos Aires. Los criterios de inclusión fueron: estar hospitalizados durante la fecha del estudio y estar tomando medicamentos, los últimos 15 días antes de ser hospitalizados.

Para la selección de pacientes, se adoptó el muestreo no probabilístico consecutivo, ingresando al estudio los pacientes hospitalizados en la fechas de realización del estudio. En el proyecto original, se planificó seleccionar hospitales pertenecientes al SNSS Argentino, en el área de Buenos Aires; sin embargo, las coordinaciones para este objetivo no tuvieron resultados positivos, por consiguiente, se decidió la inclusión de los siguientes hospitales: Monte Grande, Pilar, Clínicas y Oncológico Mari Curi, dichos centros no pertenecían al SNSS (Sistema Nacional del Seguro de Salud).

La razón principal de dicha selección fue: identificar las características de la población más vulnerable que perteneciendo al SNSS no tuvieron acceso a dichos servicios y tuvieron que acudir a los centros 
descritos. Por consiguiente, para la interpretación y análisis del presente estudio, se tuvo presente este sesgo de selección.

Se usó un cuestionario estructurado, entrevistando directamente a los pacientes capaces de responder, en el caso de los niños o adultos mayores con trastorno cognitivo o del sensorio, la entrevista fue respondida por la madre o cuidador/a respectivamente.

La recolección de información sobre fármacos tuvo en cuenta la forma de obtención de medicamento (por el SNSS con descuento respectivo, comprado en farmacia privada o pagado completamente), tipo de prescriptor (médico, no médico o automedicación). También se clasificaron los medicamentos de acuerdo a la inclusión o no en la lista de fármacos del SNSS.Todos los fármacos se clasificaron por clases, tomando como modelo la Clasificación Anatómica-Terapéutica-Química de la Organización Mundial de la Salud (8).

Definición operacional de falta de accesibilidad

Se consideró como falta de acceso a medicamentos a los fármacos incluidos en el petitorio o lista de medicamentos del SNSS que tuvieron que ser comprados íntegramente por el paciente. El cuestionario elaborado fue diseñado para detectar la falta de acceso a medicamentos prescritos por el médico (para el análisis se excluyó la prescripción farmacología de otros profesionales y la automedicación) los últimos 15 días antes de la hospitalización. La pregunta específica fue: La última vez que acudió a la cita del médico, los medicamentos recetados, ifueron cubiertos por su cobertura de salud (SNSS) o tuvo que pagarlos íntegramente usted?.

Se analizó las variables demográficas (subgrupos etarios, sexo, ingreso mensual), perfil farmacológico (número de fármacos, tipos de fármacos), no accesibilidad a medicamentos prescritos por médicos y sus razones. Se utilizó el programa bioestadístico SPSS Statistics versión 17 para el análisis de los datos. Se utilizó el test de heterogeneidad X2 para evaluar las diferencias en las proporciones, mientras que para evaluar las diferencias de las medias se uso el test $\mathrm{F}$ y $\mathrm{t}$.

\section{RESULTADOS}

Los 500 pacientes incluidos en el estudio, procedieron de hospitales del área de Buenos Aires: Monte Grande, Pilar, Clínicas y Oncológico Mari Curi. La media edad fue de $47,4+18,8$ y rango de 0.1 a 89 años; 173 (34,6\%) fueron de sexo masculino y $327(65,4 \%)$ femenino. La población estudiada incluyó a 10 niños (2\%) y 91 (18.2\%) adultos mayores (>64 años). La distribución de la cobertura de los pacientes estudiados fue predominantemente perteneciente al Sistema Público con 280 (56,0\%), seguido de Obra Social $175(35,0 \%)$ y Prepago 45 (9,0\%). Tabla 1.

De los 292 pacientes que recibieron una prescripción médica 15 días antes de ser hospitalizados, 121 $(41,4 \%)$ tuvieron disponible el medicamento, de los cuales $104(31,1 \%)$ fueron entregados por el SNSS y $17(5,3 \%)$ pacientes lo obtuvieron por donaciones. De acuerdo a la definición operacional utilizada de accesibilidad a medicamentos, $171(58,6 \%)$ pacientes no tuvieron accesibilidad a medicamentos, es decir, tuvieron que comprar íntegramente sus fármacos (medicamentos recetados).

Comparando la correlación entre el tipo de cobertura y la accesibilidad, el mayor porcentaje de falta de accesibilidad fue el tipo prepago, seguido de Obra Social y sistema público. Comparando la falta de accesibilidad a medicamentos entre los hospitales estudiados, en orden decreciente figuraron: Hospital de Clínicas Universidad de Buenos Aires, Hospital Central de Pilar, Hospital Municipal de Oncología "Marie Curie", Hospital Municipal Monte Grande "Santa Marina". Tabla 2.

Al comparar las medias de ingreso mensual familiar, no hubo diferencia significativa entre los que tuvieron 0 no accesibilidad a fármacos $(\$ 579,2+274,2$ vs $\$ 781,5+1049,3)(p>0.05)$. No hubo diferencia estadísticamente significativa entre accesibilidad entre pacientes mayores de 64 años (adultos mayores) y los menores de 65 años de edad (58.7\% vs $58.1 \%$ ), tampoco hubo diferencia significativo en relación con el sexo. El tipo de cobertura con menor accesibilidad (no accesibilidad) fue el sistema Prepago mayor $85,0 \%$, comparado con los otros tipos de cobertura $(\mathrm{p}<0.05)$. Tabla 3. 
José F. Parodi, Teodoro J. Oscanoa, Miguel A. Fernández- Pastor, Maria Cecilia Garbarino,

Mauro Tomas Ghersi

Tabla 1. Características clínicas y Demográficas de la población estudiada

\begin{tabular}{ll}
$\begin{array}{l}\text { CARACTERÍSTICAS } \\
\text { DEMOGRÁFICAS }\end{array}$ & VALORES \\
& \\
Media edad (desviación típica) & $47,403 \pm 18,7862$ \\
Rango (años) & $0.1-89$ \\
\hline Grupos etáreos & \\
0 a 4 años: & \\
5 a 14 años: & $4(0,8 \%)$ \\
15 a 44 años: & $9(1,8 \%)$ \\
45 a 64 años: & $214(42,8 \%)$ \\
65 a 74 años: & $182(36,4 \%)$ \\
75 a 85 años: & $46(9,2 \%)$ \\
$>84$ años: & $40(8,0 \%)$ \\
& $5(1,0 \%)$ \\
Sexo: $n(\%)$ & \\
Masculino & \\
Femenino & \\
Raza & $173(34.6 \%)$ \\
Blanco & $327(65.4 \%)$ \\
Negro & \\
Mestizo & $485(97.0 \%)$ \\
Estado civil & $1(0.2 \%)$ \\
Soltero & $14(2.8 \%)$ \\
Casado & \\
Unido & $176(35.2 \%)$ \\
Separado & $218(43.6 \%)$ \\
& $26(5.2 \%)$ \\
\hline & $36(7.2 \%)$ \\
\hline & \\
\hline &
\end{tabular}

ingreso Mensual (n: 76)

Media (desviación típica)

$\$ 864.61+763.076$

Rango

$\$ 150-\$ 4,500$

Hospitales Encuestados en Buenos Aires

Hospital Municipal Monte Grande "Santa Marina" $112(22,4 \%)$

Hospital Central de Pilar

$122(24,4 \%)$

Hospital de Clínicas Universidad de Buenos Aires

$111(22,2 \%)$

Hospital Municipal de Oncología "Marie Curie"

$155(31,0 \%)$

Servicio de internación

Medicina interna $\quad 341(68,2 \%)$

Cirugía $\quad 125(25,0 \%)$

Pediatría $\quad 5(1,0 \%)$

Ginecología $20(4,0 \%)$

Geriatría $\quad 1(0,2 \%)$

Otros $\quad 8(1,6 \%)$

Tipo de cobertura

Obra Social

$175(35,0 \%)$

Prepago

Sistema Público
$45(9,0 \%)$

$280(56,0 \%)$
Tabla 2. Características sobre accesibilidad y perfil farmacológico de la población estudiada

\section{CARACTERÍSTICAS}

DEMOGRÁFICAS

\section{VALORES}

Consumo de medicamentos en los últimos

15 días antes de hospitalización

$\mathrm{Si} \quad 341(68.2 \%)$
No
$159(31.8 \%)$

Origen de la prescripción de medicamentos (N:341)

Recetados por el médico

$292(85,6 \%)$

Recetados profesional no médico $\quad 7(2,1 \%)$

Automedicados $\quad 42(12,3 \%)$

Media desviación típica de número de medicamentos

(prescripción médica) por paciente ( $\mathrm{n}: 292) \quad 2,7 \pm 1,7$

Rango 1 a 9

Acceso a medicamentos prescritos por médicos ( $\mathrm{n}: 292)$

Adquiridos en el SNSS con descuento $\quad 105(36,0 \%)$

Tuvo que pagar integramente el costo $\quad 171(58,6 \%)$

Le fueron donados $\quad 16(5,5 \%)$

Método de información sobre medicamentos (N:341)

De memoria $\quad 321(94,1 \%)$

Trajo una lista $\quad 16(4.7 \%)$

Trajo los medicamentos $\quad 4(1,2 \%)$

Razones de no accesibilidad a los mericamentos

en las Farmacias de SNSS (razones por las que tuvo

que pagar integramente el fármaco) $(\mathrm{n}: 171)$

$\begin{array}{ll}\text { No había en la farmacia del SNSS } & 30(17,5 \%) \\ \text { Porque habia mucha cola } & 12(7,0 \%) \\ \text { Otros } & 129(75,5 \%)\end{array}$

Tiempo utilizado en conseguir los medicamentos

Media y desviación típica (días)

$1,97 \pm 3,6$

Rango (días)

$1-30$

Media de gasto por sus medicamentos (Dólares

americanos/últimos 15 días antes de ser hospitalizado).

Media y desviación típica

$\$ 110.98$

Rango

$\$ 2.0-1500$

Tiempo total uso fármaco

Media (años)

$3.67 \pm 6.25$

Rango

2,2 días a 20,3 años 
Tabla 3. Descripción de la población estudiada, subgrupos y accesibilidad a medicamentos

\begin{tabular}{|c|c|c|c|c|}
\hline Característica & $N(\%)$ & Uso Fármacos (\%) & $\begin{array}{l}\text { Número fármacos usados } \\
\text { (media y desviación típica) }\end{array}$ & $\begin{array}{l}\text { No acceso a medicamentos } \\
\text { (Comprados con pago } \\
\text { en su totalidad) (\%) }\end{array}$ \\
\hline Sexo & & $P<0.001$ & $P=0.11$ & $P=0.072$ \\
\hline Masculino & $173(34,6 \%)$ & 56.1 & $2.26 \pm 1.64$ & $51.8 \%$ \\
\hline Femenino & $327(65,4 \%)$ & 74.6 & $2.57 \pm 1.62$ & $61.2 \%$ \\
\hline Edad & & $P<0.001$ & $P=0.11$ & $P=0.462$ \\
\hline 0-64 años & $91(18,2 \%)$ & $64.1 \%$ & $2.1 \pm 1.56$ & $58,7 \%$ \\
\hline 65 años & $409(81,8 \%)$ & $86.8 \%$ & $3.2 \pm 1.85$ & $58,1 \%$ \\
\hline Tipo de cobertura & & $P<0.05$ & $P<0.05$ & $P<0.05$ \\
\hline Obra Social & $175(35,0 \%)$ & $74,9 \%$ & $2,67 \pm 1,82$ & $58,3 \%$ \\
\hline Prepago & $45(9,0 \%)$ & 57,8 & $2,11 \pm 1,82$ & $85,0 \%$ \\
\hline Sistema Público & $280(56 \%)$ & $65,7 \%$ & $2,09 \pm 1,54$ & $55,4 \%$ \\
\hline
\end{tabular}

La accesibilidad estudiada bajo la perspectiva del fármaco prescrito, debe observarse que se excluyó a los fármacos no incluidos en la lista de medicamentos del SNSS $(21 \%)$, de acuerdo a la definición operacional del presente estudio. No se tuvo accesibilidad a $51.5 \%$ de los fármacos prescritos incluidos en la lista de medicamentos del SNSS, del total de este porcentaje correspondió a pacientes que pertenecían a Obra Social: $43.7 \%$.

Las principales razones por las cuales los pacientes no lograron conseguir los medicamentos prescritos, fueron que, "no había" en la farmacia del SNSS $(17,5 \%)$, "había mucha cola" (7,0\%). El 75.5\% de los pacientes, esbozó razones distintas a las enumeradas en el cuestionario, algunas de las más frecuentes fueron: "cada vez que los necesito tengo que llevar la receta con todo autorizado y siempre surge algo como que no estoy en el sistema", "la obra social no se hace cargo de los medicamentos y cada vez que los necesito es un problema", "la receta le faltan datos y tengo volver a rehacerla", "Ias recetas me las tiene que hacer mi médico de cabecera y no lo encuentro frecuentemente", "no me hacían el reintegro por la obra social entonces me retenían el medicamento", "no me las daban por ser en ese momento paciente ambulatorio", "algunos los autorizan otros no entonces quedan las recetas y van de acá para allá", "no reconocen a los medicamentos como habilitados para vender con descuento", "no reconocen todos los medicamentos que necesita el paciente", "PAMI no hace descuento para este medicamento y es muy caro", "por trámite burocrático", entre otros.

La media del tiempo utilizado por los pacientes para conseguir sus medicamentos fue de $2.0+3,6$ (rango: 1 a 30 días). La media del monto gastado por el paciente en la compra de sus medicamentos fue de $\$ 110.98+226.3$ (rango: $\$ 2.0-1500.0$ )en los últimos 15 días.

Los 192 pacientes que recibieron prescripción médica de fármacos, tuvieron una media de 2,7 +1,7 de medicamentos por paciente (rango: 1 a 9).

Globalmente recibieron 769 medicamentos. Los adultos mayores (edad $>64$ años) recibieron mayor número de medicamentos que los otros grupos etarios $(p<0.001)$. 
Los 5 grupos farmacológicos (según la clasificación ATC) más frecuentemente recetados fueron en orden decreciente: sistema cardiovascular (30\%), sistema músculoesquelético $(18.7 \%)$, sistema digestivo y metabolismo (13.2\%), sistema nervioso (10.9\%) y agentes antineoplásicos (7.2\%).

El porcentaje de Medicamentos obtenidos con descuentos o pagados íntegramente en paciente perteneciente al SNSS (Obra Social), observándose que los fármacos clasificados como del sistema respiratorio, aparato genitourinario y hormonas sexuales y sistema musculoesquelético son lo que tienen menos acceso.

\section{DISCUSIÓN}

El presente estudio, encontró una falta de accesibilidad a medicamentos del $58,6 \%$. Entre las razones para la no accesibilidad a medicamentos la mayoría (75.5\%) atribuyó a múltiples razones pero que pueden ser descritas como errores en los procesos de distribución del fármaco. Cuando los pacientes consiguieron sus medicamentos, la media del tiempo utilizada para este fin fue de 2 días (rango 1 a 30 días). La media del gasto por la compra de sus fármacos en los últimos 15 días fue \$111. (rango: \$2 a 1500). De acuerdo a la cobertura de atención de salud, la no accesibilidad a medicamentos fue mayor en el sistema Prepago $(89.5 \%)$ y Obra social (58.3\%). El estudio de utilización de medicamentos encontró una media de media de 2,7 de medicamentos por paciente (rango: 1 a 9 ).

Los estudios de accesibilidad de medicamentos pueden llevarse a cabo de dos maneras, de acuerdo a la población investigada: mediante encuesta a hogares o con estudios en población usuaria de servicio de salud. El presente estudio es de esta última población. Bertoldi y col, realizaron un estudio en encuesta a hogares en Porto Alegre, Brasil, encontrando falta de acceso en 3.6\%, 41.5\% pagaron totalmente sus medicamentos $(25 \%$ de familias muy pobres) y $43.2 \%$ refirió como causa "porque pensé (o sabía) que esos medicamentos no tenían en el Programa Salud Familiar", Ios medicamentos de este programa se distribuyen gratuitamente (9).
En Regiones Sur y Noreste del Brasil en encuesta a hogares, se encontró falta de acceso a medicamentos de uso continuo (antihipertensivos, antidiabéticos orales y fármacos para trastornos mentales) en adultos y adultos mayores de 19 y $23 \%$ respectivamente (10). El principal factor predictor de falta de acceso en este último estudio fue la ausencia de vínculo con programas nacionales (Unidades Básicas de Salud, Programa de Hipertensión Arterial y Diabetes Mellitus) y trastornos mentales. Para efectos de comparación con otros estudios similares, es muy importante aclarar que la mayor parte de las investigaciones sobre accesibilidad de medicamentos en Latinoamérica han sido con encuesta a hogares, por consiguiente las comparaciones que se hacen deben ser tomadas en ese contexto, puesto que los estudios de accesibilidad en usuarios de servicios de salud se caracterizan por ser población altamente seleccionados, pacientes crónicos y con enfermedades más severas. La importancia de hacer este tipo de estudios en usuarios de servicios de salud, es focalizar los principales problemas de accesibilidad para diseñar estudios de intervención inmediata que puedan mejorar las deficiencias a esta población vulnerable.

El presente estudio, presentó algunas limitaciones como que el diseño original del estudio fue estudiar la accesibilidad a medicamentos en pacientes hospitalizados en centros hospitalarios pertenecientes al SNSS, sin embargo, por diversas razones no se logró autorización para dicho estudio en las diversas clínicas o centros privados contratados por el SNSS. Las características de la población estudiada: población usuaria de servicios de salud, excluida voluntaria o involuntariamente de asistir a centros específicos del SNSS, medicación 15 días antes de ser hospitalizada (incluye medicación para enfermedades agudas y/o crónicas). Los hospitales seleccionados no pertenecen al SNSS, sin embargo, atienden pacientes pertenecientes al SNSS. La población estudiada tuvo un sesgo de selección de pacientes con falta de accesibilidad al sistema que se quiso estudiar. Sin embargo, sus hallazgos pueden reflejar las características de una población especialmente vulnerable y que requiere urgentemente medidas de intervención para aumentar su accesibilidad no solamente a los servicios de salud del SNSS, sino también a los medicamentos. 
El diseño de la población estudiada, con usuarios de servicios de salud, habitualmente son personas que acuden por enfermedades agudas, o crónicas reagudizadas, por consiguiente sus hallazgos no pueden extrapolarse a la población en general; se requieren hacer estudios con encuestas a hogares para comprobar los hallazgos descritos.

Además, la comparación con otros estudios que hayan utilizado la misma definición operacional o similar instrumento, no es factible, porque los estudios sobre este tópico se caracterizan por no tener hasta el momento un instrumento de referencia o validados (11), aunque debe señalarse que posteriores al presente estudio se han hecho propuestas muy interesantes al respecto (12).

La definición operacional utilizada excluyó medicamentos no incluidos en la lista de medicamento de la SNSS: por lo tanto, las cifras de no accesibilidad descritas podrían incrementarse, si se consideran también los fármacos excluidos en dicha lista de fármacos del SNSS, aunque debe aclararse que la mayor parte de estos fármacos son de bajo valor terapéutico (Ejemplo:condroitin sulfato).

En conclusión, no se encontró falta de accesibilidad a medicamentos los últimos 15 días antes de su hospitalización del 58.6\%. Las razones de la no accesibilidad aparentemente son por errores en los procesos que se inician desde le emisión de las recetas hasta la no disponibilidad de fármacos en las farmacias de los hospitales estudiados pertenecientes al SNSSA.

\section{Fuentes de financiamiento}

Convenio de la Superintendencia de Servicios de Salud y el Centro Interamericano de Estudios de Seguridad Social (CIESS).

\section{Conflictos de interés}

Los autores declaran no tener conflictos de interés. Correspondencia:

J osé F. Parodi

Dirección: Av. Alameda del Corregidor 1531-

Las Viñas, La Molina. Lima - Perú.

Teléfono: 51-13652300 / 4422275 / 998139858.

Correo electrónico: jparodig@usmp.pe

\section{REFERENCIAS BIBLIOGRÁFICAS}

1. Leyva-Flores R, Erviti-Erice J, Kageyama-Escobar ML, Arredondo A.Prescripción, acceso y gasto en medicamentos entre usuarios de servicios de salud en México. Salud pública de México 1998; 40(1):24-31.

2. Laporte J-R, Tognoni G. Estudios de utilización de medicamentos y de farmacovigilancia. En: JR Laporte, G Tognoni Eds. Principios de epidemiología del medicamento. $2^{a}$ ed. Barcelona. Ediciones Científicas y Técnicas, 1993: 2-23.

3. Portal Oficial del Gobierno de la República de Argentina [En línea] // Sistema de Salud Argentino: Empresas. - 12 de Diciembre de 2009.

4. Ronconi L. La seguridad social en Argentina [En línea]. - Centro de Estudios para el Desarrollo Institucional - Fundación Gobierno y Sociedad, Marzo de 2002. - 14 de Diciembre de 2009. -http:// www.monografias.com/trabajos913/seguridad-social-argentina/ seguridad-social-argentina. pdf.

5. Montoya S, Colina J. La Reforma de Obras Sociales en Argentina: Avances y Desafíos Pendientes [Informe] / Procesos de Reforma en Educación Social y Salud. Diseño, Implementación y Grupos de Interés; Centro Internacional de Investigaciones para el Desarrollo (IDRC/CIID), Banco Interamericano de Desarrollo (IDB/BID). 1998.

6. Findling $L$, López $E$, Abramzón $M$, Lehner $P$, Ponce $M$, Venturiello M. Desigualdades en salud y redes sociales. Una aproximación a la situación de los adultos mayores [Conferencia] // Jornada. VII Jornadas Nacionales de Debate Interdisciplinario en Salud y Población. 2007 / ed. UBA Área de Salud y Población - Instituto de Investigaciones Gino Germani -. Facultad de Ciencias Económicas UBA - Buenos Aires : [s.n.], 2007.

7. Zerda A. Sistemas de seguros de salud y acceso a medicamentos Estudios de casos de Argentina, Colombia, Costa Rica, Chile, Estados Unidos de América y Guatemala [Libro]. - [s.l.]: Pan American HealthOrganization, 2001. - Artes y Letras, Buenos Aires: pág. 99. ISBN 9275323534 .

8. ATC/DDD Index 2009. [En línea] // WHO Collaborating Centre for Drug Statistics Methodology. - http:// www. whocc. no/ atcddd/ .

9. Bertoldi $A D$, de Barros AJ, Wagner A, Ross-Degnan D, Hallal PC: Medicine access and utilization in a population covered by primary health care in Brazil. Health Policy 2009, 89(3):295-302.

10. Paniz VM, Fassa AG, Facchini LA, Bertoldi AD, Piccini RX, Tomasi E, Thume E,Silva da Silveira D, Siqueira FV, Rodrigues MA: Access to continuous-use medication among adults and the elderly in South and Northeast Brazil. Cad SaudePublica 2008; 24(2): 267-280.

11. Paniz VM, Fassa AG, Maia MF, Domingues MR, Bertoldi AD. Measuring access to medicines: a review of quantitative methods used in house hold surveys. BMC Health Serv Res 2010;10:146.

12. Oscanoa TJ.[Access and usability to medications: a proposal for an operational definition]. Rev Peru Med Exp Salud Publica 2012 Mar; 29(1):119-26.
Recibido: 09 de Diciembre de 2013 Aprobado: 10 de Febrero de 2014 